\title{
Omics provide insights to understand cotton responsive to Verticillium dahliae and application (Abstract)
}

\author{
Longfu Zhu \\ College of Plant Science and Technology, Huazhong Agricultural University, Wuhan, China \\ Corresponding author: lfzhu@ mail.hzau.edu.cn
}

\begin{abstract}
Due to no high resistant germplasm in upland cotton (Gossypium hirsutum), Verticillium wilt has become the most serious disease for cotton production in China. Through whole genome gene expression analysis by RNA sequence, we found the expression level of lignin synthesis-related genes and the activity of corresponding enzymes induced more quickly in resistant cultivar '7124' (G. barbasense) [1]. Recently, an ethylene responserelated factor, GbERF1-like, from '7124' was proved as a positive regulator in lignin synthesis and contributed substantially to resistance to $V$. dahliae [2]. And suppression the expression level of a cotton P450 gene, SILENCE-INDUCED STEM NECROSIS (SSN), causes a lesion mimic phenotype in cotton. Further study shows that SSN silencing causes an imbalance in LOX (lipoxygenase) expression and excessive hydroperoxide fatty acid accumulation. We also show that an unknown oxylipin-derived factor is a putative mobile signal required for systemic cell death [3]. Complex phytohormones interaction was also found involving in cotton responsive to $V$. dahliae [4]. BR and JA signal pathways play essential roles in interaction of cotton and $V$. dahliae [4, 5]. Furthermore, we identify the key data from genomics and proteomics with a data-mining strategy accompanied by VIGS and heterologous expression [6]. GbWRKY1 is one of the key candidate genes and has been proved with as a critical regulator mediating the plant defense-to-development transition during $V$. dahliae infection by activating JAZ1 expression [7]. Overall, our study provides highlights to understand the mechanism of cotton resistance to $V$. dahliae at molecular level.
\end{abstract}

Keywords: Omics, cotton, verticillium dahliae. 
